\title{
Análise comparativa do perfil epidemiológico do câncer de pele não-melanoma no Brasil, Nordeste e Maranhão, no período 2015-2019
}

Comparative analysis of the epidemiological profile of non-melanoma skin cancer in Brazil, Northeast and Maranhão, in the 2015-2019 period

Análisis comparativo del perfil epidemiológico del cáncer de piel no-melanoma en Brasil, Noreste y Maranhão, en el período 2015-2019

\author{
Yasmine Araújo Victor \\ ORCID: https://orcid.org/0000-0003-1197-9528 \\ Universidade Federal do Maranhão, Brasil \\ E-mail: yasmine.a.victor@gmail.com \\ Izolda Souza Costa \\ ORCID: https://orcid.org/0000-0003-0899-6845 \\ Universidade Federal do Maranhão, Brasil \\ E-mail: izolda.costa@hotmail.com \\ Nilson de Jesus Pereira Batalha Júnior \\ ORCID: https://orcid.org/0000-0002-6325-5655 \\ Universidade Federal do Maranhão, Brasil \\ E-mail: nilsonbatalha@gmail.com \\ Sueli de Souza Costa \\ ORCID: https://orcid.org/0000-0003-4127-7324 \\ Universidade Federal do Maranhão, Brasil \\ E-mail: scsueli@gmail.com \\ Cristianne Talitha Lopes Sampaio \\ ORCID: https://orcid.org/0000-0001-8372-3236 \\ Universidade Federal do Maranhão, Brasil \\ E-mail: tianne_talitha@hotmail.com \\ Luísa Fernanda Pires Campos \\ ORCID: https://orcid.org/0000-0002-2086-2578 \\ Universidade Federal do Maranhão, Brasil \\ E-mail: luisacampospires@hotmail.com
}

\begin{abstract}
Resumo
O acúmulo de mutações que resulta no crescimento desordenado de células é denominado câncer. Tal doença atinge milhares de pessoas no mundo e seu principal tipo é o de pele de origem não melanocítica, que inclui os carcionomas basocelular e espinocelular. Seu principal fator de risco é a exposição aos raios solares de forma desprotegida. Assim, sendo o Brasil um país de clima majoritariamente tropical, recebe uma quantidade de radiação significativa, tornando seus residentes mais suscetíveis aos danos de uma fotoexposição prolongada, principalmente na região nordeste. $\mathrm{O}$ objetivo deste trabalho foi realizar uma análise epidemiológica dos últimos cinco anos no Maranhão, no Nordeste e no Brasil sobre o câncer de pele não-melanoma, através de estudo observacional analítico transversal, quali-quantitativo, sendo que foram coletados dados do painel de oncologia disponível no DATASUS no período de 2015 a 2019. Os resultados demonstraram que o câncer de pele é o mais incidente e prevalente no Brasil, principalmente na população igual e maior que 70 anos, sendo o Rio Grande do Sul o estado mais acometido do Brasil e o Rio Grande do Norte, o do Nordeste. É mais prevalente na população feminina. A campanha de saúde "Dezembro Laranja" é hoje a principal estratégia de prevenção ao câncer de pele no Brasil.
\end{abstract}

Palavras-chave: Neoplasias cutâneas; Carcinoma basocelular; Carcinoma espinocelular; Epidemiologia.

\begin{abstract}
The accumulation of mutations that results in disordered cell growth is called cancer. This disease affects thousands of people worldwide and its main type is skin of non-melanocytic origin, which includes basocellular and spinocellular carcionomas. Its main risk factor is unprotected exposure to sunlight. Thus, since Brazil is a country with a mostly tropical climate, it receives a significant amount of radiation, making its residents more susceptible to the damage of a prolonged photoexposure, especially in the northeast region. The objective of this work was to carry out an epidemiological analysis of the last five years in Maranhão, in the Northeast and in Brazil on non-melanoma skin cancer, through observational analytical cross-sectional study, qualitative and quantitative, and data were collected
\end{abstract}


from the available oncology panel at DATASUS in the period from 2015 to 2019. The results showed that skin cancer is the most incident and prevalent in Brazil, mainly in the population equal and older than 70 years, with Rio Grande do Sul being the most affected state in Brazil and the Rio Grande do Norte, the Northeast. It is more prevalent in the female population. The health campaign "December Orange" is today the main strategy for the prevention of skin cancer in Brazil.

Keywords: Skin cancer; Basal cell skin cancer; Squamous cell skin cancer; Epidemiology.

\section{Resumen}

La acumulación de mutaciones que resulta en un crecimiento celular desordenado se llama cáncer. Esta enfermedad afecta a miles de personas en todo el mundo y su principal tipo es la piel de origen no melanocítico, que incluye carcionomas basocelulares y espinocelulares. Su principal factor de riesgo es la exposición al sol sin protección. Así, dado que Brasil es un país con un clima mayoritariamente tropical, recibe una cantidad significativa de radiación, lo que hace que sus habitantes sean más susceptibles a los daños de una fotoexposición prolongada, principalmente en la región noreste. El objetivo de este trabajo fue realizar un análisis epidemiológico de los últimos cinco años en Maranhão, en el Nordeste y en Brasil sobre el cáncer de piel no-melanoma, a través de un estudio observacional analítico transversal, cualitativo y cuantitativo, y se recolectaron datos de el panel de oncología disponible en DATASUS en el período de 2015 a 2019. Los resultados mostraron que el cáncer de piel es el más incidente y prevalente en Brasil, principalmente en la población igual y mayor de 70 años, siendo Rio Grande do Sul el estado más afectado en Brasil y el Rio Grande do Norte, el Nordeste. Es más frecuente en la población femenina. La campaña de salud "Diciembre Naranja" es hoy la principal estrategia para la prevención del cáncer de piel en Brasil.

Palabras clave: Neoplasias cutáneas; Carcinoma de células basales; Carcinoma de células escamosas; Epidemiología.

\section{Introdução}

Câncer é o nome dado a um conjunto de mais de 100 doenças que têm em comum o crescimento desordenado de células, que invadem tecidos e órgãos. Sua etiologia é multifatorial e resulta, principalmente, de alterações genéticas, fatores ambientais e comportamentais (INCA, 2020). Dentre os fatores ambientais encontra-se a exposição exacerbada aos raios ultravioleta (UV), como os emitidos pelo sol, especialmente sobre a pele, apesar da importância que raios UV possuem na saúde humana, ao possibilitar a síntese de vitamina D (Oliveira, 2013).

O câncer de pele corresponde a $33 \%$ de todos os diagnósticos de tumores malignos no Brasil. A cada ano são encontrados cerca de 180 mil novos casos de câncer de pele, sendo a maioria não-melanoma do tipo carcinoma basocelular, apresentando incidência de cerca de 70\%. Ele é formado nas células basais da epiderme, apresenta-se com menor agressividade, tem crescimento lento, raramente desenvolve metástase e as áreas mais comuns de se encontrar são face e regiões fotoexpostas (Bernardes, 2016).

O segundo tipo mais comum de não-melanoma é o carcinoma espinocelular cuja incidência é de $25 \%$, possui maior capacidade de produzir metástase linfática e visceral, sendo que o tumor se desenvolve na camada espinhosa da pele (acima da camada basal) e as áreas mais comumente encontradas são face, orelha, nariz, pescoço, dorso das mãos e mucosas oral e genital; destacando-se o fato de lesões de pele preexistentes, como a ceratose actínica, serem fatores facilitadores do surgimento do carcinoma espinocelular. Ambos os tipos mais comuns de não-melanoma possuem bom prognóstico quando detectados precocemente (Da Silva \& Dias, 2017).

O aumento na incidência do câncer de pele foi significativo nos últimos 30 anos, destacando-se alguns fatores para esse ocorrido: mudança nos hábitos de vida com exposição solar demasiada em horários diversos, por trabalho ou lazer; envelhecimento populacional; desenvolvimento de técnicas diagnósticas e sua consequente detecção precoce; rarefação da camada de ozônio; residir em país tropical (Pires et al., 2017). No que tange às características pessoais, há algumas que predispõem o surgimento de neoplasias cutâneas: cor da pele, olhos e cabelos claros; presença de sardas e nevos; história pessoal ou antecedentes familiares de câncer de pele; uso de imunossupressão crônica (Imanichi, Moraes, Soteroi, \& Gomes, 2017).

A radiação ultravioleta do sol (UV) é um potente agente carcinógeno. Possui os subtipos UVA, UVB e UVC de 
acordo com o comprimento de onda. Os raios UVA apresentam o comprimento de onda mais longo (315-400nm), indutores de processos oxidativos, como o envelhecimento. A banda UVB (280-315nm) é responsável por danos diretos ao DNA, fotoimunossupressão, eritema, espessamento do estrato córneo e melanogênese. Os raios UVC (100-280nm) são carcinogênicos e contêm o pico de absorção pelo DNA puro (Silva et al., 2015; Bechara, 2015). A exposição demasiada ao sol, sem proteção e principalmente por pessoas de pele clara, que tem um tipo de melanina diferente dos que possuem pele escura e são mais protegidos, é um fator preocupante. Isso principalmente em áreas onde a radiação ultravioleta chega com mais facilidade e intensidade, como a região Nordeste do Brasil (Da Silva \& Dias, 2017).

A região Nordeste do Brasil apresenta um vasto litoral onde a atividade turística é fortemente empregada, principalmente o turismo do tipo "sol e mar". Como o próprio nome já denuncia, a exposição solar nesta região do país é altamente endossada, fazendo com que ela apresente um dos maiores índices de câncer de pele no Brasil. Somado a isso, é essencial destacar que a tal região apresenta em sua climatologia radiação UV do tipo eritêmica ou biologicamente ativa com valores considerados altos (Lopo, Spyrides, Lucio, \& Sigró, 2013; Linos, Katz, \& Colditz, 2016). A estimativa de novos casos das neoplasias de pele para 2020, é de 185.380 incluindo melanoma e não-melanoma em homens e mulheres (Imanichi et al., 2017; Guerra et al., 2017).

No Brasil, é a região Sul a que concentra maior número de câncer de pele não-melanoma (CPNM), embora seja a região geográfica com menores índice de radiação ultravioleta (Imanichi et al, 2017). As regiões Nordeste (40,37/ 100 mil) e Norte (28,34/ 100 mil) ocupam a segunda posição no ranking de incidência de CPNM, entre os homens, e entre as mulheres é o mais frequente em todas as regiões do país, sendo que a região Nordeste apresentou taxa de 46,68/ 100 mil em 2014 (Oliveira, 2013). Dos cânceres de pele, 176.940 são do tipo não-melanoma sendo 37380 esperados para a região Nordeste, e 2.290 no estado do Maranhão, em 2020 (INCA, 2019). Em 2015, no Brasil, o câncer de pele melanoma (CPM) foi responsável por 1012 mortes em homens e 782 em mulheres; e o não-melanoma cursou com 1137 óbitos na população masculina contra 821 na feminina (INCA, 2019).

A negligência em torno da ocorrência de câncer da pele ainda é uma realidade no Brasil, sendo que, somada ao atraso no diagnóstico ou conduta de tratamento, exacerba os riscos de recidiva, metástase e óbito (Espósito et al., 2017).

A região Nordeste sofre grande influência da radiação solar sobre a região equatorial, e o estado do Maranhão, por se encontrar na região geográfica litorânea do Nordeste, é um local onde poderia haver maior quantidade de câncer de pele. Analisar o tipo de câncer de pele é fundamental para direcionar políticas públicas de saúde, a fim de minimizar o problema, especialmente quando se trata do tipo não-melanoma, pois este possui bom prognóstico quando detectado precocemente (Da Silva \& Dias, 2017).

Assim, o objetivo deste trabalho é realizar uma análise epidemiológica dos últimos cinco anos no Maranhão, no Nordeste e no Brasil sobre o câncer de pele não-melanoma, a fim de contribuir com o conhecimento científico, bem como servir de Norte para políticas públicas de saúde.

\section{Metodologia}

Trata-se de um estudo observacional analítico transversal, quali-quantitativo (Pereira, Shitsuka, Parreira, \& Shitsuka, 2018), onde foram coletados dados do painel de oncologia disponível no DATASUS no período de 2015 a 2019 sobre os diagnósticos gerais de câncer e do câncer de pele não-melanoma no Brasil, Nordeste e Maranhão.

Foram incluídos na pesquisa como elementos fixos: a unidade federativa dos diagnósticos, tanto de câncer geral quanto de câncer de pele não-melanoma, e o período de 2015 a 2019; e dentre as variáveis estão: sexo e faixa etária.

Foram feitas tabelas no programa Microsoft Excel $^{\circledR}$ nas seguintes apresentações: número de diagnósticos de câncer de 
2015 a 2019 em todo o Brasil, na região Nordeste e no estado do Maranhão, divididos por sexo e faixa etária em cada ano referido no período. Dentro desses números foram comparados os diagnósticos de câncer de pele não-melanoma notificados no período de tempo apresentado, divididos nas variáveis citadas (faixa etária e sexo) e nos locais expostos.

A partir desses dados, foi realizada uma análise gráfica no programa Microsoft Excel ${ }^{\circledR}$ traçando um perfil epidemiológico do câncer mais prevalente do Brasil, qual ano de maior acometimento, qual faixa etária de maior prevalência e qual sexo mais acometido.

Por serem informações de livre acesso e circulação, não houve necessidade de submissão ao Comitê de Ética em Pesquisa, de acordo com a Resolução 466/2012 do Conselho Nacional de Saúde, do Ministério da Saúde.

\section{Resultados}

O número de diagnósticos de câncer no Brasil manteve-se quase uma constante de 2015 a 2017. A partir desse último ano, houve um significativo crescimento nos anos seguintes sendo de $84 \%$ em 2018, e de 66\% em 2019, como é mostrado na Tabela 1.

Tabela 1. Números de câncer gerais em valores absolutos no Maranhão, Nordeste e Brasil, no período de 2015 a 2019.

\begin{tabular}{cccccc} 
& $\mathbf{2 0 1 5}$ & $\mathbf{2 0 1 6}$ & $\mathbf{2 0 1 7}$ & $\mathbf{2 0 1 8}$ & $\mathbf{2 0 1 9}$ \\
\hline & 2.791 & 3.031 & 3.070 & 5.354 & 11.444 \\
Maranhão & 36.027 & 38.038 & 38.326 & 77.734 & 123.235 \\
Nordeste & 165.772 & 169.619 & 168.856 & 311.333 & 518.413 \\
Brasil & &
\end{tabular}

Fonte: Painel de Oncologia, DATASUS, 2015-2019.

De 2015 a 2017 a diferença no número de diagnósticos de homens e mulheres assim como a diferença na quantidade de casos entre os sexos foi pouco significativa. A partir de 2018, o número de casos tanto femininos quanto masculinos cresceu em maior proporção, de acordo com a Tabela 2.

Tabela 2. Números absolutos de diagnósticos de câncer geral e casos de câncer de pele não-melanoma divididos por sexo no Maranhão, Nordeste e Brasil, no período de 2015 a 2019.

\begin{tabular}{|c|c|c|c|c|c|}
\hline \multirow{3}{*}{ Ano } & \multirow{3}{*}{$\begin{array}{c}\text { Estado/ } \\
\text { Região/ } \\
\text { País }\end{array}$} & \multicolumn{4}{|c|}{ Sexo } \\
\hline & & \multicolumn{2}{|c|}{ Masculino } & \multicolumn{2}{|c|}{ Feminino } \\
\hline & & CG & CPNM & CG & CPNM \\
\hline \multirow{3}{*}{2015} & MA & 1.209 & 9 & 1.580 & 7 \\
\hline & NE & 16.580 & 308 & 19.430 & 233 \\
\hline & BR & 80.591 & 1.368 & 85.145 & 1.187 \\
\hline \multirow{3}{*}{2016} & MA & 1.314 & 14 & 1.714 & 3 \\
\hline & NE & 17.180 & 328 & 20.835 & 216 \\
\hline & BR & 81.464 & 1.344 & 88.096 & 1.344 \\
\hline
\end{tabular}


Continuação Tabela 2. Números absolutos de diagnósticos de câncer geral e casos de câncer de pele não-melanoma divididos por sexo no Maranhão, Nordeste e Brasil, no período de 2015 a 2019.

\begin{tabular}{|c|c|c|c|c|c|}
\hline \multirow{3}{*}{ Ano } & \multirow{3}{*}{$\begin{array}{l}\text { Estado/ } \\
\text { Região/ } \\
\text { País }\end{array}$} & \multicolumn{4}{|c|}{ Sexo } \\
\hline & & \multicolumn{2}{|c|}{ Masculino } & \multicolumn{2}{|c|}{ Feminino } \\
\hline & & CG & CPNM & CG & CPNM \\
\hline \multirow{3}{*}{2017} & MA & 1.318 & 8 & 1.752 & 12 \\
\hline & $\mathbf{N E}$ & 17.468 & 275 & 20.832 & 258 \\
\hline & BR & 81.268 & 1.229 & 87.524 & 1.086 \\
\hline \multirow{3}{*}{2018} & MA & 2.322 & 263 & 3.032 & 241 \\
\hline & $\mathbf{N E}$ & 33.173 & 2.369 & 44.561 & 2.479 \\
\hline & BR & 145.858 & 12.955 & 165.232 & 13.648 \\
\hline \multirow{3}{*}{2019} & MA & 4.509 & 352 & 6.935 & 353 \\
\hline & $\mathbf{N E}$ & 49.145 & 3.771 & 74.090 & 4.023 \\
\hline & BR & 229.787 & 27.909 & 287.845 & 31.021 \\
\hline
\end{tabular}

Legenda: MA = Maranhão; NE = Nordeste; $\mathrm{BR}=$ Brasil $;$ CG = câncer geral; CPNM = câncer de pele não-melanoma. Fonte: Painel de Oncologia, DATASUS, 2015-2019.

Os estados do Ceará, Bahia e Pernambuco são campeões em diagnósticos de câncer em todos os anos analisados, como mostra o Gráfico 1.

Gráfico 1. Diagnósticos de câncer divididos por estado do Nordeste de 2015 a 2019.

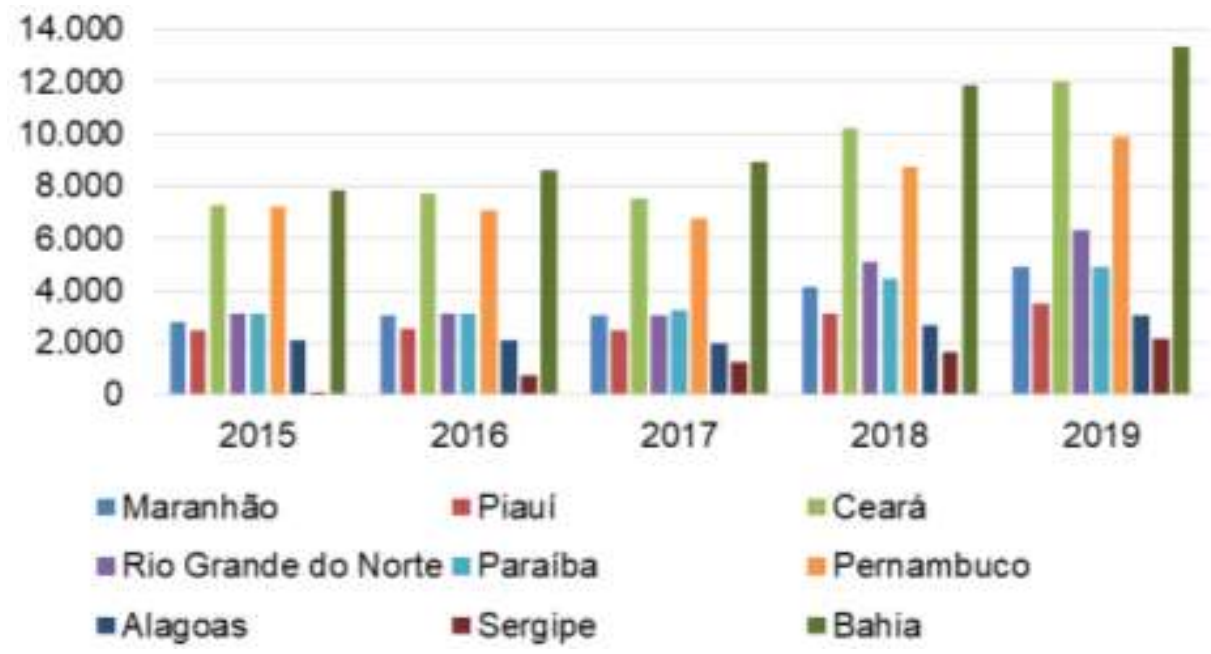

Fonte: Painel de Oncologia, DATASUS, 2015-2019.

O mesmo fenômeno de quase constância nos diagnósticos de 2015 a 2017 foi observado. A partir de 2018 houve um significativo salto, assim como em 2019. No Maranhão, por exemplo, de 2017 para 2018 houve um aumento de 2.420\%, percebido no Gráfico 2. 
Gráfico 2. Diagnósticos de câncer de pele não-melanoma divididos por ano e por estados da região Nordeste do Brasil no período de 2015 a 2019.

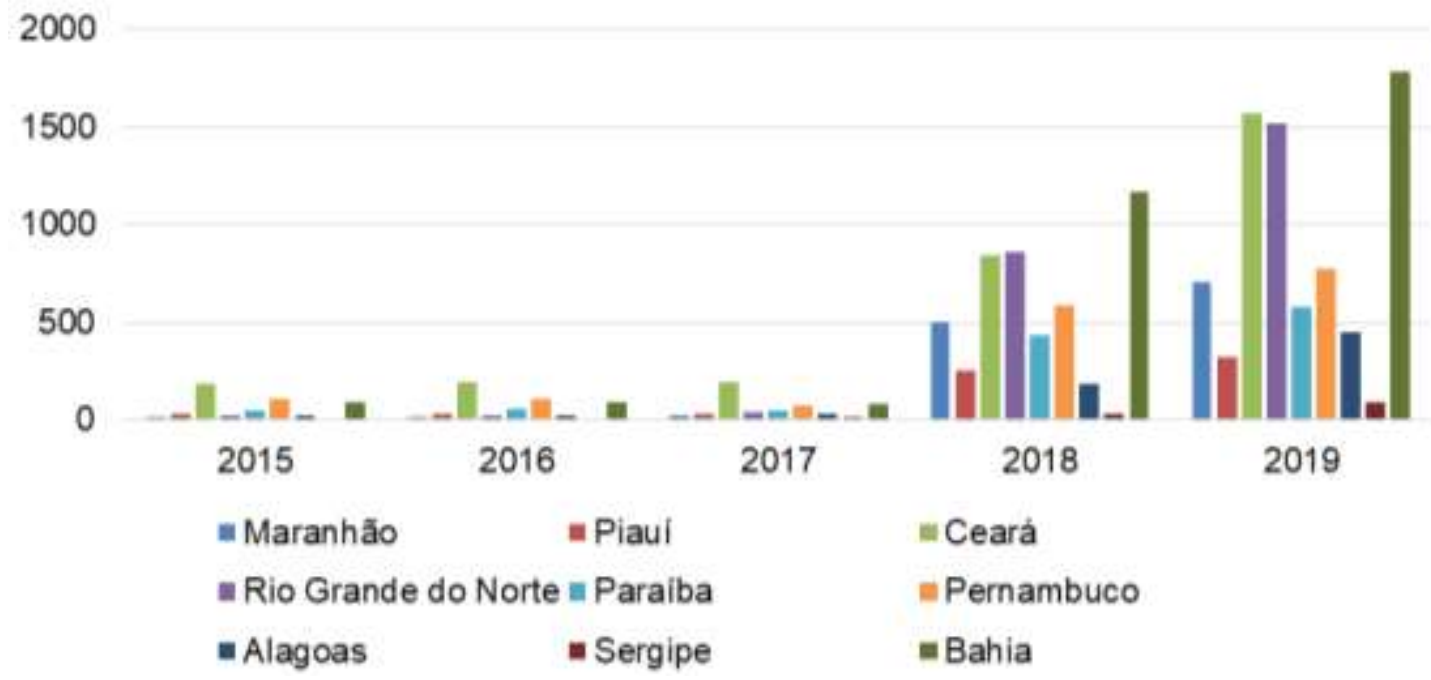

Fonte: Painel de Oncologia, DATASUS, 2015-2019.

A faixa etária 55-69 anos é a que possui maior número de diagnósticos de câncer em todos os anos analisados. De 2017 para 2018 houve um aumento de $75 \%$ no número de casos e, de 2018 para 2019 foi de $61 \%$ nessa faixa etária, como demontrado no Gráfico 3.

Gráfico 3. Diagnósticos de câncer por faixa etária, de 2015 a 2019.

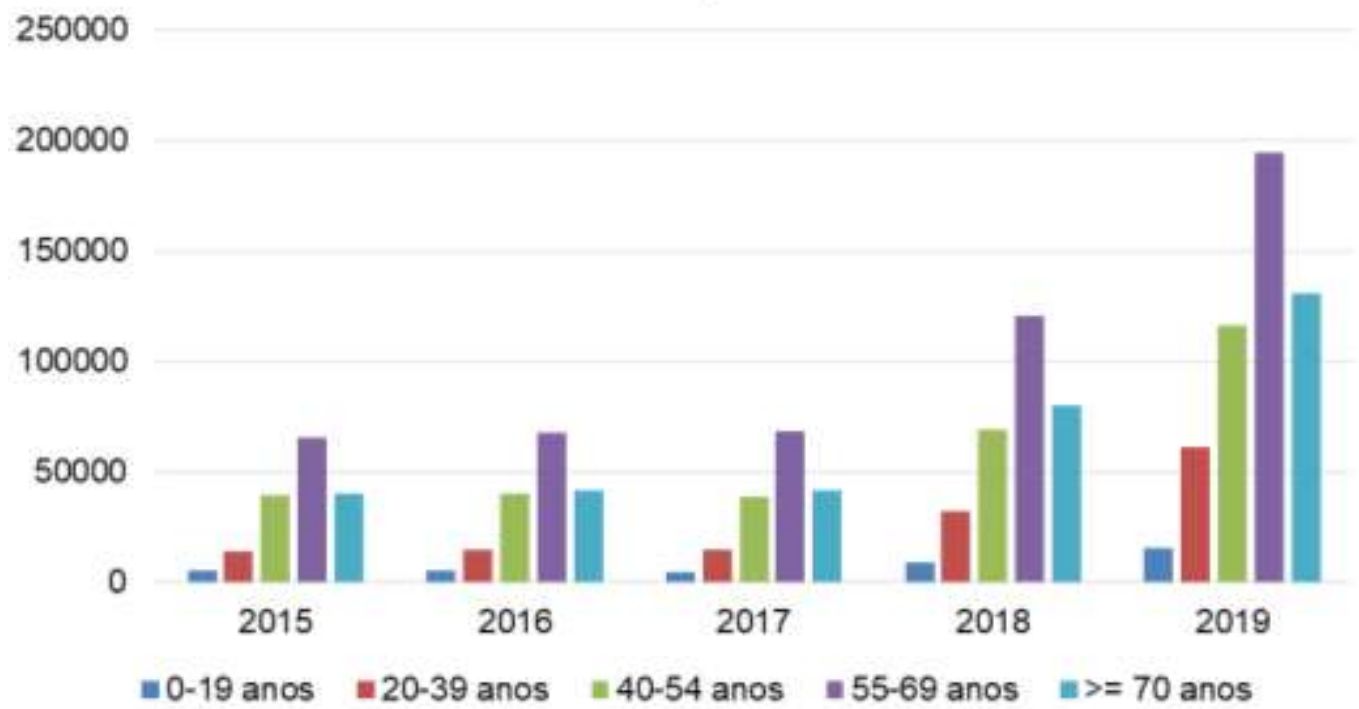

Fonte: Painel de Oncologia, DATASUS, 2015-2019.

Indivíduos com idade maior ou igual a 70 anos são os campeões em diagnósticos de câncer de pele não-melanoma em todos os anos analisados. Vale destacar também o significativo aumento nesta faixa etária de 2017 para 2018, correspondendo a $859 \%$, e de 2018 para 2019 de $108 \%$, como segue no Gráfico 4. 
Gráfico 4. Diagnósticos de câncer de pele não-melanoma por faixa etária no Brasil, no período de 2015 a 2019.

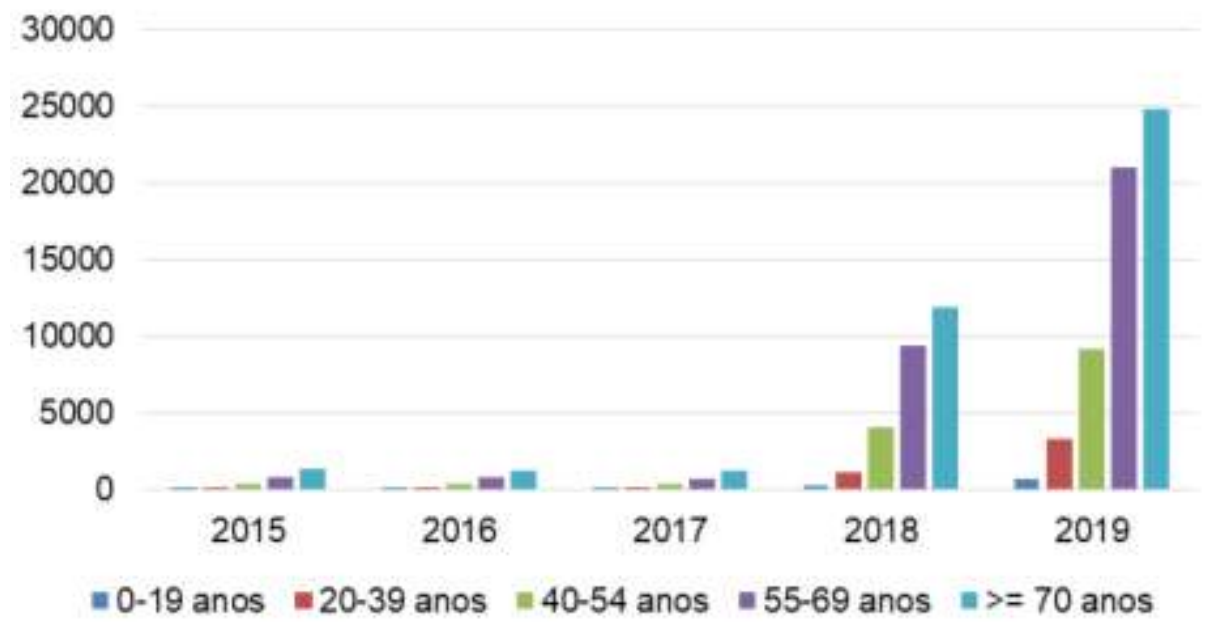

Fonte: Painel de Oncologia, DATASUS, 2015-2019.

Os números de câncer no período de 2015 a 2017 mostraram-se quase numa constante, com variáveis pouco significativas, a nível de Brasil, Nordeste e Maranhão. De 2017 para 2018, os diagnósticos de câncer no Brasil cresceram em cerca de $84 \%$, e de 2018 a 2019 foi de $66 \%$.

Esse significativo aumento ocorreu também com os números de câncer de pele não-melanoma. No intervalo de 2017 para 2018 houve um salto de 150\% e, de 2018 para 2019, 121\%. Em 2017, esse tipo de neoplasia correspondia a 1,37\% dos diagnósticos totais de câncer no Brasil. Já em 2018, a porcentagem foi para 8,57\% e, em 2019, 11,39\% do total de 147 diagnósticos específicos catalogados pelo Painel de Oncologia do DATASUS. No Nordeste, o crescimento de casos de CPNM no ano de 2018 foi de 809\% em relação ano anterior; em 2019 o aumento diminuiu para 60\% com relação a 2018. No Maranhão, de 2017 para 2018 o aumento de números de casos de CPNM foi de 2420\%; de 2018 para 2019, foi de 109\%.

O sexo masculino e o feminino apresentaram variações pouco expressivas de 2015 a 2017 de números de diagnósticos de câncer e de CPNM, tanto no Maranhão, quanto no Nordeste e no Brasil. De 2017 para 2018, no Brasil, o número de neoplasias cresceu em 79\% nos homens e 88\% nas mulheres; de 2018 para 2019 o aumento foi de 57\% e 74\%, respectivamente, sendo nas mulheres o maior número de casos. O CPNM representou de 2015 a 2017, em ambos os sexos 1 a 2\% dos diagnósticos gerais de câncer no Brasil. Já em 2018, o CPNM representou 8,2\% dos diagnósticos de câncer no sexo feminino e $8,8 \%$ no sexo masculino; em 2019, a representação foi de $12,1 \%$ para o sexo masculino e $10,7 \%$ para o feminino. Na população masculina, o aumento percentual de casos de CPNM, no Brasil, foi de 954\% em 2018 e $115 \%$ em 2019; no Nordeste, em 2018, foi de 761\% e em 2019 foi 115\%; no Maranhão foi 3.187\% em 2018 e 33\% em 2019. Na população feminina, por sua vez, o aumento de casos de CPNM foi de $1.160 \%$ no Brasil, $860 \%$ no Nordeste e $1.908 \%$ no Maranhão em 2018. Já em 2019, 126\% no Brasil, 62\% no Nordeste e 46\% no Maranhão. Em números de casos absolutos, o sexo feminino apresenta o maior número de casos.

O estado mais acometido, em valores absolutos, tanto pelo câncer quanto pelo CPNM, é o estado de São Paulo, por se tratar do estado mais populoso do Brasil. No entanto, em 2019, o número de casos/100.000 habitantes foi de 37 para o CPNM. O estado do Brasil com maior número de casos/100.000 habitantes é o Rio Grande do Sul com 73, seguido do Mato Grosso do Sul com 72 casos / 100.000 habitantes. Realizando um comparativo com alguns dos estados mais populosos do Nordeste como Bahia e Ceará, que se destacaram em prevalência naquele ano, os números de casos/100.000 habitantes foram 12,7 e 18,5, respectivamente. O estado do Rio Grande do Norte, o $16^{\circ}$ mais populoso do Brasil, por sua vez, apresentou o maior número de 
casos/100.000 habitantes do Nordeste, num total de 48/ 100.000 habitantes, destacando-se em relação à São Paulo. Já o Maranhão, em 2019, apresentou uma taxa de 10,7 casos por 100.000 habitantes.

Dentre as faixas etárias analisadas, a campeã em diagnósticos de câncer no geral é a de 55 a 59 anos em todos os anos estudados. No que tange ao CPNM a faixa etária maior ou igual a 70 anos é a que ganha maior destaque, tanto em números absolutos, quanto em prevalência. Em 2018, a faixa etária de 55 a 59 anos somou um total de 120.513 diagnósticos de câncer, destes 9.424 eram de CPNM, que corresponde a 7,8\%; em 2019, dos 194.957 casos, 21.047 ou 10,8\% correspondiam ao CPNM. Na faixa etária de maior ou igual a 70 anos, no ano de 2018, dos 80.241 diagnósticos de câncer, 11.897 corresponderam ao CPNM, o que representa 14,8\%; em 2019, dos 130.555 diagnósticos de câncer, 24.828 foram de CPNM, correspondente a 19,02\%. Dos 58.928 diagnósticos de CPNM no Brasil em 2019, 35\% deles está na faixa etária de 55 a 59 anos e $40 \%$ deles está na população igual ou maior que 70 anos. Segundo o Instituto Brasileiro de Geografia e Estatística (IBGE), em 2019 o Brasil contava com 19.625.301pessoas com idade entre 55 e 69 anos, delas 21.047 tiveram CPNM, o que significa 107 casos a cada 100.000 pessoas; no mesmo ano havia 9.240.224 pessoas com 70 anos ou mais, delas 24.828 tiveram CPNM, o que significa 268 casos a cada 100.000 habitantes dessa faixa etária.

\section{Discussão}

O significativo aumento do número de casos tanto de câncer no geral, quanto do câncer de pele não-melanoma de 2017 para 2018 e de 2018 para 2019 é, sobretudo, um marco do melhor monitoramento dos casos de câncer no Brasil, outrora subnotificados. A partir de 2018, o Instituto Nacional do Câncer José de Alencar Gomes da Silva (INCA), por meio da Divisão de Detecção Precoce desenvolveu junto com o Departamento de Informática do Sistema Único de Saúde (DATASUS) a plataforma do Painel de Oncologia, que permitiu o fornecimento de uma base de dados melhor elaborada, antes incipientemente feita pelos Sistemas de Informações em Saúde (SIS) no Sistema Único de Saúde (SUS) (Atty, Jardim, Dias, Migowski, \& Tomazelli, 2020).

O melhor panorama da situação do câncer no Brasil passou a ocorrer a partir de 2018. O crescente número de casos percebidos a partir desse ano é retrato da transição demográfica ocorrida no Brasil, na qual o número de idosos vem aumentando e, por conseguinte, mudanças no perfil epidemiológico direcionado para doenças crônicas não transmissíveis, como o câncer (Bomfim, Giotto, \& Silva, 2018). As faixas-etárias mais acometidas são de 55 a 69 anos e os iguais/acima de 70 anos, sendo esta última a mais prevalente no número de casos de câncer de pele não-melanoma. O maior acúmulo de mutações causado pelo fotodano tende a ser mais evidente quanto maior for a idade, por isso o CPM e o CPNM aumentam suas incidências com o aumento das faixas etárias (Wright, du Preez, Millar, \& Norval, 2020).

O estado de São Paulo atualmente é o campeão em número de casos de 2015 a 2019, tanto de diagnósticos gerais de câncer, quanto dos de pele não-melanoma. Ele conta com uma população de 46 milhões de pessoas, sendo o mais populoso do Brasil (IBGE, 2020). Isso, por sua vez, torna-se um fator determinante quando comparado a outros estados do Brasil. Contudo, o Rio Grande do Sul (RS), que tem um total de 10.693.929 habitantes, apresentou um total de 8.342 casos de CPNM no ano de 2019, deixando o estado à frente aos demais do Brasil em casos a cada 100.000 habitantes. Tal estado possui a maior quantidade de pessoas brancas do Brasil, cerca de $83 \%$ da população, o que corrobora um dos principais fatores de risco para o desenvolvimento do CPNM: ter os fototipos de pele I (sempre queima, nunca bronzeia) e II (queima facilmente, bronzeia minimamente) (Barton et al., 2017).

Esse fenômeno ocorre também no Nordeste, com o estado da Bahia, sendo o mais populoso da região e o mais prevalente nos números de câncer. No entanto, o cenário muda em relação ao CPNM: de 2018 a 2019, o Rio Grande do Norte (RN), um dos menos populosos do Nordeste apresentou as maiores taxas de incidência e prevalência desse tipo de neoplasia. 
No último censo do IBGE, o RN tinha uma população de 3.168.027, sendo $41 \%$ declarados brancos. Comparado ao Ceará e à Bahia, que possuem $31 \%$ e $22 \%$ de indivíduos brancos, respectivamente. Além de possuir o turismo de sol e mar como uma das atividades econômicas mais importantes do estado (Coutinho, 2015). Isso fomenta dois fatores de risco importantes para o surgimento das neoplasias de pele, incluindo o CPNM, o tipo de pele e a maior exposição aos raios solares.

O Maranhão apresentou no último censo, uma população de aproximadamente 6,5 milhões de pessoas. Dessas, 22\% são brancas. Em 2019, dos 11.444 diagnósticos de câncer, 9,2\% eram de CPNM, número este abaixo da média brasileira de 11,3\% do total de diagnósticos e acima da média do Nordeste, que foi de 6,3\%. No entanto, tanto no Maranhão, quanto em todo o Nordeste, o CPNM fica em $2^{\circ}$ lugar em números absolutos de casos, perdendo apenas para o câncer de próstata.

No Brasil, a população feminina representa cerca de 51,8\% dos brasileiros. Isso é igual ao um total de 97.348.809, contra 93.406.990 da população masculina. Os 31.021 casos de CPNM em 2019 representam 31,8 casos/ 100.000 mulheres; na população masculina, seus 27.909 casos representam 29,8 casos/ 100.000 homens. Percebe-se que não há uma diferença muito significativa de acometimento das neoplasias de pele não-melanoma entre homens e mulheres, apesar delas apresentarem mais casos. No entanto, o segundo tipo mais comum de CPNM, o carcinoma de células escamosas, tende a atingir duas vezes mais os homens quando comparado às mulheres (Soares, Mendes, \& Sampaio, 2021).

Quanto aos tipos de câncer de pele não-melanoma, tanto o INCA quanto o Painel de Oncologia do DATASUS não especificam em seus dados estatísticos quais os mais prevalentes. No entanto, o carcinoma basocelular corresponde a $70 \%$ dos diagnósticos dos não-melanomas, sendo o carcinoma de células escamosas ou espinocelular o segundo tipo mais comum (Oliveira, 2013).

Mesmo com altas taxas de incidência e prevalência, principalmente na faixa etária acima de 70 anos, a mortalidade desse câncer comparado aos demais de grande incidência também, como o de próstata em homens e o de mama em mulheres, é muito baixa (Carvalho \& Paes, 2019). Apesar disto, no país, a maioria dos casos de câncer aponta taxas de mortalidade crescentes, exceto os de estômago e de colo do útero que estão com taxas em declínio. Ademais, a literatura destaca que as taxas de mortalidade por câncer, apontam tendência de aumento nas regiões Norte e Nordeste, no período 2011-2030, sendo que as demais regiões tenderão à estabilidade ou decréscimo (Sousa et al., 2016; Guerra et al., 2017; Azevedo, Bezerra-Rocha, Fernandes, \& Veríssimo-Fernandes, 2019; Barbosa, Souza, Bernal, \& Costa, 2019; Soares, Mendes, \& Sampaio, 2021).

O câncer de pele no Brasil é um problema de saúde pública, sendo o mais incidente e prevalente nacionalmente, e esta situação aponta a necessidade de estruturação dos sistemas de saúde para que esteja apto a lidar com custos altos de diagnósticos e tratamento, bem como propiciar prevenção e rastreamento de tais casos (Guerra et al., 2017; Soares, Mendes, \& Sampaio, 2021).

A partir disso, foi desenvolvido em 2014 uma Campanha Nacional de Prevenção ao Câncer de Pele pela Sociedade Brasileira de Dermatologia designada de "Dezembro Laranja", visando promover um mês inteiro de educação em saúde voltado para a população. A campanha conta com forte apelo às mídias sociais, apresentando aos indivíduos os principais fatores de risco e como evitar os possíveis, a exemplo da exposição solar. Relata ainda quando suspeitar de alguma lesão de pele, fornecendo as principais características sugestivas de malignidade (SBD, 2018).

Por mais que tal iniciativa já tenha 7 anos, os resultados só serão percebidos a longo prazo, devido à fisiopatologia do câncer de pele, que é resultado de um acúmulo de danos ao DNA da pele ao longo de vários anos (Montagna \& Lopes, 2017). Por isso, é importante que a campanha seja feita na base da população, para que a cultura de fotoproteção seja instaurada nas práticas sociais a curto, médio e longo prazos. Sendo assim, a edição do ano de 2020 do Dezembro Laranja contou com o apoio dos maiores influenciadores digitais infantis do Brasil para deixá-la mais difundida e gerar educação em saúde de base. Além disso, já que o câncer de pele é altamente prevenível fazendo uso de filtro solar com fator de proteção igual ou maior a 30, as instituições governamentais poderiam democratizar o acesso a esses produtos na atenção primária de maneira ampla e gratuita, 
onde o cuidado com a população é iniciado.

\section{Conclusão}

O câncer de pele não-melanoma é a neoplasia de maior incidência e prevalência em todo o território do Brasil. Dentro dele, há dois tipos principais que respondem a mais de 90\% dos diagnósticos desse tipo de câncer, o carcinoma basocelular, cuja representatividade é $70 \%$ dos casos, e o carcinoma espinocelular ou de células escamosas. O estado brasileiro mais acometido é o Rio Grande do Sul, já que é composto por maioria caucasiana.

No Nordeste e no Maranhão, o CPNM ganha em incidência, mas perde em prevalência para o câncer de próstata, sendo o Rio Grande do Norte o estado que apresenta maior incidência e prevalência. O CPNM acomete um pouco mais mulheres, porém o carcinoma de células escamosas é mais prevalente na população masculina brasileira. A faixa etária mais acometida no Brasil é correspondente aos indivíduos com 70 anos ou mais.

Esta pesquisa demonstra que o câncer de pele é um problema de saúde pública, sendo passível de diagnóstico precoce e cuidados preventivos, devendo haver ações como educação em saúde, visando minimizar gastos desnecessários e melhorar a qualidade de vida das pessoas.

Em virtude da importância dos dados demonstrados, sugere-se que periodicamente novas pesquisas sobre a temática venham a público com intuito de atualização constante dos levantamentos, favorecendo difusão técnico-científica e ações de políticas públicas.

\section{Referências}

Atty, A. T. D. M., Jardim, B. C., Dias, M. B. K., Migowski, A., \& Tomazelli, J. G. (2020). PAINEL-Oncologia: uma Ferramenta de Gestão. Revista Brasileira de Cancerologia, 1-10.

Azevedo, P. R. M., Bezerra-Rocha, J., Fernandes, T. A. A. M., \& Veríssimo-Fernandes, J. (2019). Analysis of cervical cancer mortality rate trends in NatalRN, Brazil, between 2000 and 2012. Revista de Salud Pública, 21, 161-167.

Barbosa, I. R., Souza, D. L. B. D., Bernal, M. M., \& Costa, I. D. C. C. (2016). Desigualdades regionais na mortalidade por câncer de colo de útero no Brasil: tendências e projeções até o ano 2030. Ciência \& Saúde Coletiva, 21, 253-262.

Barton, V., Armeson, K., Hampras, S., Ferris, L. K., Visvanathan, K., Rollison, D., \& Alberg, A. J. (2017). Nonmelanoma skin cancer and risk of all-cause and cancer-related mortality: a systematic review. Archives of dermatological research, 309(4), 243-251.

Bechara, E. J. H. (2015). Sol, Melanina e Câncer: o Bom, o Mau e o Feio. Revista Virtual de Química, 7(4), 1565-1569.

Bernardes, A. V. (2016). Prevenção do câncer de pele em trabalhadores do setor agrícola. Revista Pró-UniverSUS, 7(3), 03-07.

Bomfim, S. S., Giotto, A. C., \& Silva, A. G. (2018). Câncer de pele: conhecendo e prevenindo a população. Revista de Divulgação Científica Sena Aires, 7(3), 255-259.

Carvalho, J. B., \& Paes, N. A. (2019). Corrected cancer mortality rates for the elderly in the states of the Brazilian Northeast. Ciencia \& saude coletiva, 24(10), 3857-3866.

Coutinho, A. C. A. (2015). Políticas públicas, desenvolvimento local e participação social nas instâncias de governança associadas ao turismo no Rio Grande do Norte. Dissertação de Mestrado, Universidade Federal do Rio Grande do Norte.

da Silva, R. D., \& Dias, M. A. I. (2017). Incidência do carcinoma basocelular e espinocelular em usuários atendidos em um hospital de câncer. Revista Família, Ciclos de Vida e Saúde no Contexto Social, 5(2), 228-234.

Espósito, A. C. C., Campos, E. B. P., Marques, M. E. A., Marques, A. S., Abbade, L. P. F., \& Stolf, H. O. (2017). Fatores que levam a negligência quanto aos canceres de pele não melanoma. Diagnóstico e tratamento, 22(2), 63-6.

Guerra, M. R., Bustamante-Teixeira, M. T., Corrêa, C. S. L., Abreu, D. M. X. D., Curado, M. P., Mooney, M., Naghavi, M., Teixeira, R., França, E. B., \& Malta, D. C. (2017). Magnitude e variação da carga da mortalidade por câncer no Brasil e Unidades da Federação, 1990 e 2015 . Revista Brasileira de Epidemiologia, 20, 102-115.

Imanichi, D., Moraes, C. F., Soteroi, R. D. C., \& Gomes, L. O. (2017). Fatores de risco do câncer de pele não melanoma em idosos no Brasil. Diagnóstico e tratamento, 22(1), 3-7.

Instituto Brasileiro de Geografia e Estatística (IBGE) (2020). Cidades e Estados do Brasil <https://cidades.ibge.gov.br/>. 
Research, Society and Development, v. 10, n. 5, e14410514552, 2021

(CC BY 4.0) | ISSN 2525-3409 | DOI: http://dx.doi.org/10.33448/rsd-v10i5.14552

Instituto Nacional de Câncer José Alencar Gomes da Silva (INCA) (2020). Ministério da Saúde. O que é Câncer. <https://www.inca.gov.br/o-que-e-cancer> .

Instituto Nacional de Câncer José Alencar Gomes da Silva (INCA) (2019). Ministério da Saúde. Estimativa 2020: Incidência de Câncer no Brasil. <https://www.inca.gov.br/sites/ufu.sti.inca.local/files//media/document//estimativa-2020-incidencia-de-cancer-no-brasil.pdf>.

Linos, E., Katz, K. A., \& Colditz, G. A. (2016). Skin cancer-the importance of prevention. JAMA internal medicine, 176(10), 1435-1436.

Lopo, A. B., Spyrides, M. H. C., Lucio, P. S., \& Sigró, J. (2013). Radiação ultravioleta, ozônio total e aerossóis na cidade de Natal-RN. Holos, 6, 3-21.

Montagna, E., \& Lopes, O. S. (2017). Molecular basis of basal cell carcinoma. Anais brasileiros de dermatologia, 92(4), 517-520.

Oliveira, M. M. F. (2013). Radiação ultravioleta/índice ultravioleta e câncer de pele no Brasil: condições ambientais e vulnerabilidades sociais. Revista Brasileira de Climatologia, 13.

Pereira, A. S., Shitsuka, D. M., Parreira, F. J., \& Shitsuka, R. (2018). Metodologia da pesquisa científica. 〈http://repositorio.ufsm.br/bitstream/handle/1/15824/Lic_Computacao_Metodologia-Pesquisa-Cientifica.pdf?sequence=1〉.

Pires, C. A. A., Fayal, A. P., Cavalcante, R. H., Fayal, S. P., Lopes, N. S., Fayal, F. P., \& dos Santos, M. A. L. (2017). Câncer de pele: caracterização do perfil e avaliação da proteção solar dos pacientes atendidos em serviço universitário. Journal of Health \& Biological Sciences, 6(1), 54-59.

Silva, A. L. A., Sousa, K. R. F., Silva, A. F., Fernandes, A. B. F., Matias, V. L., \& Colares, A. V. (2015). A importância do uso de protetores solares na prevenção do fotoenvelhecimento e câncer de pele. Revista Interfaces: Saúde, Humanas e Tecnologia, 2(7).

Soares, L. S., Mendes, A. C. S., \& Sampaio, J. R. F. (2021). Incidência e mortalidade das neoplasias malignas na região Nordeste/Brasil no período de 1979 a 2016: uma Revisão Integrativa. Brazilian Journal of Development, 7(3), 33262-33275.

Sociedade Brasileira de Dermatologia (SBD) (2018). Câncer da Pele. <https://www.sbd.org.br/dermatologia/pele/doencas-e-problemas/cancer-da-pele/64/> .

Sousa, A. M. V. D., Teixeira, C. C. A., Medeiros, S. D. S., Nunes, S. J. C., Salvador, P. T. C. D. O., Barros, R. M. B. D., Lima, F. F. S., Nascimento, G. G. C., Santos, J., Souza, D. L. B., Bezerra, A. P. S., \& Meira, K. C. (2016). Mortalidade por câncer do colo do útero no estado do Rio Grande do Norte, no período de 1996 a 2010: tendência temporal e projeções até 2030. Epidemiologia e Serviços de Saúde, 25, 311-322.

Wright, C. Y., du Preez, D. J., Millar, D. A., \& Norval, M. (2020). The epidemiology of skin cancer and public health strategies for its prevention in Southern Africa. International journal of environmental research and public health, 17(3), 1017. 